\title{
ON SECTIONAL GENUS OF $k$-VERY AMPLE LINE BUNDLES ON SMOOTH SURFACES WITH NON-NEGATIVE KODAIRA DIMENSION
}

\author{
YOSHIAKI FUKUMA*
}

\begin{abstract}
Let $(X, L)$ be a polarized surface over the complex number field. Assume that $L$ is $k$-very ample. In this paper, we study the relation between the sectional genus $g(L)$ and the irregularity $q(X)$. In particular we prove $g(L) \geq(k+2) q(X)$ if $X$ has the Kodaira dimension $\kappa(X)=0,1$, or $(X, L)$ is some special cases with $\kappa(X)=2$. Moreover we classify $(X, L)$ with $g(L)=(k+2) q(X)$ when $\kappa(X)=0$ or 1 .
\end{abstract}

\section{§0. Introduction}

Let $X$ be a smooth projective manifold over the complex number field with $\operatorname{dim} X=n \geq 2$ and let $L$ be an ample line bundle on $X$. Then we call $(X, L)$ a polarized manifold. The sectional genus of $(X, L)$ is defined by the following formula:

$$
g(L):=1+\frac{1}{2}\left(K_{X}+(n-1) L\right) L^{n-1},
$$

where $K_{X}$ is the canonical divisor of $X$.

In [Fk1] and [Fk2], we studied the relation between the sectional genus and the irregularity of $X$. In particular, we considered the following Conjecture in the case of $\operatorname{dim} X=2$.

CONJECTURE 1. Let $(X, L)$ be a polarized manifold. Then $g(L) \geq q(X)$, where $q(X)$ is the irregularity of $X$.

It is not known whether this Conjecture is true or not even if $\operatorname{dim} X=2$. But if $L$ is ample and spanned, this Conjecture is true.

In this paper, we consider the case in which $X$ is a smooth projective surface with $\kappa(X) \geq 0$ and $L$ is $k$-very ample (see Definition 1.1). In this case, we propose the following Conjecture about sectional genus:

1991 Mathematics Subject Classification. Prımary 14C20.

Key words and phrases. Polarized surface, sectional genus, $k$-very ample.

* Research Fellow of the Japan Society for the Promotion of Science

Received October 7, 1997; revised February 16, 1998. 
CONJeCture 2. Let $(X, L)$ be a polarized surface. Assume that $L$ is $k$-very ample and $\kappa(X) \geq 0$. Then $g(L) \geq(k+2) q(X)$.

If $L$ is ample but not spanned, then we put $k:=-1$. Then Conjecture 2 is considered as a generalization of Conjecture 1 when $X$ is a surface with $\kappa(X) \geq 0$. In this paper, we consider the case in which $k \geq 0$ and we will prove Conjecture 2 if $(X, L)$ is one of the following cases:

(1) The case in which $\kappa(X)=0$ and $k \geq 0$ (see Section 2),

(2) The case in which $\kappa(X)=1$ and $k \geq 0$ (see Section 3),

(3) The case in which $(X, L)$ is one of the special cases with $\kappa(X)=2$ (see Section 4).

Furthermore if $\kappa(X)=0$ or 1 , then we will classify $(X, L)$ with $g(L)=$ $(k+2) q(X)$.

In general, the inequality $g(L) \geq(k+2) q(X)$ is not true if $\kappa(X)=-\infty$ and $L$ is $k$-very ample. In Appendix, we consider a lower bound for sectional genus of $k$-very ample line bundle with $\kappa(X)=-\infty$.

In this paper we work over the complex number field and we use the customary notation in algebraic geometry.

The author would like to express his gratitude to the referee for giving him many valuable comments and suggestions.

\section{§1. Preliminaries}

Denition 1.1 (See [BeSo1] or [BeSo2]). Let $(X, L)$ be a polarized surface. Then $L$ is called $k$-very ample for a nonnegative integer $k$ if for any 0 -dimensional subscheme $\left(Z, \mathcal{O}_{Z}\right)$ with length $\mathcal{O}_{Z} \leq k+1$, the map

$$
\Gamma(L) \rightarrow \Gamma\left(L \otimes \mathcal{O}_{Z}\right)
$$

is surjective.

THEOREM 1.2 (Fujita). Let $(X, L)$ be a polarized manifold with $\operatorname{dim} X=$ $n \geq 2$ and let $\Delta(L):=n+L^{n}-h^{0}(L)$ be the delta genus of $(X, L)$.

(1) $\Delta(L) \geq 0$. If $\Delta(L)=0$, then $\kappa(X)=-\infty$ and $q(X)=0$.

(2) If $\mathrm{Bs}|L|=\emptyset, \quad g(L) \geq \Delta(L)$, and $L^{n} \geq 2 \Delta(L)+1$, then $g(L)=\Delta(L)$, $\kappa(X)=-\infty, L$ is very ample, and $q(X)=0$.

(3) If $X$ is a smooth surface with $\kappa(X) \geq 1$, Bs $|L|=\emptyset, g(L)>\Delta(L)$, and $L^{2}=2 \Delta(L)$, then $q(X)=0$.

Proof. (1) See (1.4.2) and (1.5.10) in [Fj2].

(2) See (1.3.5) in [Fj2].

(3) By assumption and Theorem 1.4 in $[\mathrm{Fj} 1],(X, L)$ is a hyperelliptic polarized manifold. So by $(6.1)$ in $[\mathrm{Fj} 1]$ we get $q(X)=0$.

Proposition 1.3. (1) Let $X$ be a smooth projective variety with $\operatorname{dim} X=$ $n \geq 2$ and let $\pi: X \rightarrow \boldsymbol{P}^{n}$ be a double covering. Then $q(X)=0$. 
(2) Let $X$ be a smooth projective surface and let $L$ be an ample and spanned line bundle on $X$. If $L^{2} \leq 2$, then $q(X)=0$.

Proof. (1) See Theorem 1 in [La].

(2) Since $L$ is ample and spanned, we get $h^{0}(L) \geq 3$. Hence we get $\Delta(L) \leq 1$. If $\Delta(L)=1$, then $L^{2}=2$ and $h^{0}(L)=3$. Therefore there exists a double covering $\pi: X \rightarrow P^{2}$ defined by $|L|$. By $(1)$, we get $q(X)=0$.

If $\Delta(L)=0$, then by Theorem 1.2 we have $q(X)=0$.

Proposition 1.4. Let $X$ be a smooth projective surface and let $\pi: X \rightarrow \boldsymbol{P}^{2}$ be a triple covering defined by an ample and spanned line bundle L. Let $\mathscr{E}$ be a vector bundle of rank two on $\boldsymbol{P}^{2}$ such that $\pi_{*}\left(\mathcal{O}_{X}\right)=\mathcal{O}_{\boldsymbol{P}^{2}} \oplus \mathscr{E}$ and let $c_{2}:=c_{2}(\mathscr{E})$. Then the following hold:

(1) $\chi\left(\mathcal{O}_{X}\right)=(1 / 2) g(L)(g(L)+1)+2-c_{2}$,

(2) $K_{X}^{2}=2 g(L)^{2}-4 g(L)+11-3 c_{2}$.

Proof. See Lemma 3.2 in [Bes].

LEMmA 1.5. Let $(X, L)$ be a polarized surface with $\kappa(X) \geq 0$. Assume that $L$ is spanned and $g(L) \leq 2$. Then $q(X)=0$.

Proof. Since $\kappa(X) \geq 0$, we get $L^{2} \leq 2$. By Proposition 1.3 (2), we get $q(X)=0$.

Proposition 1.6. Let $X$ be a smooth projective surface which is embedded by a very ample line bundle $L$ in $P^{4}$. Then

$$
L^{2}\left(L^{2}-5\right)-10(g(L)-1)+12 \chi\left(\mathcal{O}_{X}\right)=2 K_{X}^{2} .
$$

Proof. See p. 434 in [Ha].

THEOREM 1.7 (Di Rocco). Let $(X, L)$ be a polarized surface with $\kappa(X) \geq 0$. If $L$ is a $k$-very ample line bundle with $L^{2} \leq 4 k+4$ and $k \geq 2$, then $X$ is a minimal K3-surface or a minimal Enriques surface.

Proof. By using the same argument as in Section 6 in [Di], it is sufficient to prove the following Claim.

ClaIm 1.7.1. Let $L$ be a k-very ample line bundle on $X$ with $k \geq 2$, $L^{2} \leq 4 k+4$, and $g(L) \leq 3 k+1$. If $\kappa(X) \neq-\infty$, then $X$ is either a minimal $K 3$ surface or a minimal Enriques surface.

Proof. Since $g(L) \leq 3 k+1$, we get $h^{1}\left(L_{C}\right) \leq 1$ by Proposition 2.5 in [Di], where $C \in|L|$ is a smooth irreducible curve. Assume that $\kappa(X) \geq 1$. Then we remark that $K_{X} L>0$. 
If $h^{1}\left(L_{C}\right)=1$, then by Theorem 2.5 in [BaSo] and the assumption we get $L^{2} \geq 2 k+g(L)$ because $K_{C} \neq L_{C}$. Since

$$
2 k+2 \geq \frac{1}{2} L^{2} \leq 2 k+\frac{1}{2} K_{X} L+1,
$$

we get $K_{X} L \leq 2$. By Theorem 4.4 in [BaSo], we get $K_{X} L \geq(k+2) / 2$. Hence $k=2$ and $K_{X} L=2$. On the other hand by Corollary 2.6 in [Di] we get $K_{X} L \leq k-1$ and this is a contradiction.

If $h^{1}\left(L_{C}\right)=0$, then $L^{2} \geq 2 k+g(L)+1$ or $L^{2} \geq k+2 g(L)$ by Lemma 2.10 in [BaSo]. If $L^{2} \geq 2 k+g(L)+1$, then

$$
2 k+2 \geq \frac{1}{2} L^{2} \geq 2 k+2+\frac{1}{2} K_{X} L
$$

and so we get $K_{X} L \leq 0$. This is a contradiction. If $L^{2} \geq k+2 g(L)$, then $-2-k \geq K_{X} L$ and this is a contradiction.

Therefore $\kappa(X)=0$. By Corollary 2.6 in [Di], we get $K_{X} L \leq k-1$. So by Theorem 4.4 in [BaSo], $X$ is minimal. Assume that $q(X) \geq 1$. Then $\chi\left(\mathcal{O}_{X}\right)=0$ by the classification theory of surfaces and we get $h^{0}(L)=L^{2} / 2$. On the other hand, by Lemma 2.8 in [BaSo], we get that $h^{0}(L) \geq 2 k+3$ and $L^{2} \geq 4 k+6$. This is a contradiction by assumption. Therefore $q(X)=0$ and $X$ is a minimal K3 surface or a minimal Enriques surface.

LEMMA 1.8. Let $(X, L)$ be a polarized surface such that $\kappa(X)=0$ and $X$ is not minimal. Let $\mu: X \rightarrow S$ be the minimalization of $X$ and let $A:=\mu_{*}(L)$ in the sense of cycle theory.

(1) Assume that $(S, A) \cong\left(E_{1} \times E_{2}, p_{1}^{*} D_{1}+p_{2}^{*} D_{2}\right)$, where $E_{l}$ is a smooth elliptic curve, $p_{i}$ is the $i$-th projection, and $D_{i} \in \operatorname{Pic}\left(E_{l}\right)$ for $i=1$ and 2 with $\operatorname{deg} D_{1}=1$ and $\operatorname{deg} D_{2} \geq 1$. Then $\operatorname{Bs}|L| \neq \emptyset$.

(2) If $\mathrm{Bs}|L|=\emptyset$ and $A^{2} \geq 6$, then $\mathrm{Bs}|A|=\emptyset$.

Proof. (1) Let $f_{2}=p_{2} \circ \mu$ and let $F_{2}$ be a general fiber of $f_{2}$. Then $F_{2}$ is a smooth elliptic curve. Since $L F_{2}=\mu^{*}(A) F_{2}=1$, we get $\mathrm{Bs}|L| \neq \emptyset$.

(2) By assumption and (1), $(S, A) \not\left(E_{1} \times E_{2}, p_{1}^{*} D_{1}+p_{2}^{*} D_{2}\right)$. Since $A^{2} \geq 6$, we get that Bs $|A|=\emptyset$ by Theorem 2.1 in [Fk5] (see also Chapter 10, $\S 1$ in [LB]).

Lemma 1.9. Let $(X, L)$ be a polarized surface. Assume that $L$ is $k$-very ample with $k \geq 0$. Then

(1) $L C \geq \max \{k, 1\}$ for any irreducible curve $C$.

(2) $L C \geq k+2$ for any irreducible curve $C$ with $C \not \boldsymbol{P}^{1}$.

(3) $L C \geq k+3$ for any irreducible curve $C$ with $g(C) \geq 2$ and $k \geq 1$.

Proof. (1) Since $L$ is ample, we get $L C \geq 1$. Hence we obtain (1) by Corollary 1.3 in [BeSol]. 
(2) If $C \not P^{1}$ and $k=0$ (resp. 1), then $L C \geq 2$ (resp. $\geq 3$ ) since $L$ is ample. If $k \geq 2$, then (2) is obtained by Proposition 1.4 in [BeSol].

(3) This can be easily obtained by (2) and Proposition 1.4 in [BeSo1].

LEMMA 1.10. Let $(X, L)$ be a polarized surface with $\kappa(X) \geq 0$ and let $f: X \rightarrow C$ be a fiber space with $g(F) \geq 1$ for a general fiber $F$ of $f$, where $C$ is $a$ smooth projective curve. (For the definition of a fiber space, see Definition 1.14 below.) Assume that $f$ is not relatively minimal and any fiber of the relatively minimal model of $f$ is smooth. If $L$ is $k$-very ample with $k \geq 0$, then $L F \geq$ $\max \{2 k+2, k+3\}$.

Proof. By assumption there exists a fiber $F$ of $f$ such that $F=F_{s}+\sum_{i} a_{i} E_{l}$, where $F_{s}$ is a smooth irreducible curve with $g\left(F_{s}\right) \geq 1$ and $E_{l}$ is a smooth rational curve. Since $L$ is ample and $k$-very ample, we get $L F_{s} \geq k+2$ and $L E_{l} \geq$ $\max \{k, 1\}$ by Lemma 1.9. Hence $L F \geq \max \{2 k+2, k+3\}$.

Lemma 1.11. Let $(X, L)$ be a polarized surface with $\kappa(X)=1$ and let $f: X \rightarrow C$ be an elliptic fibration, where $C$ is a smooth projective curve. Assume that $q(X)=g(C)+1$ and the relatively minimal model of $f$ has a multiple fiber. If $L$ is a k-very ample line bundle with $k \geq 0$, then $L F \geq 2(k+2)$, where $F$ is a general fiber of $f$.

Proof. Let $f^{\prime}: X^{\prime} \rightarrow C$ be the relatively minimal model of $f$ and let $\mu: X \rightarrow X^{\prime}$ be its birational morphism. Let $F_{1}=m F_{r}$ be a multiple fiber of $f^{\prime}$. Since $q\left(X^{\prime}\right)=q(X)=g(C)+1, F_{r}$ is a smooth elliptic curve. Let $\left(F_{r}\right)_{s}$ be the strict transform of $F_{r}$ via $\mu$. Then $L\left(F_{r}\right)_{s} \geq k+2$ by Lemma 1.9. Hence $L F=L \mu^{*}\left(F_{1}\right) \geq 2(k+2)$.

LEMMA 1.12. Let $(X, L)$ be a polarized surface with $\kappa(X)=1$ and let $f: X \rightarrow C$ be an elliptic fibration, where $C$ is a smooth curve. Let $f^{\prime}: X^{\prime} \rightarrow C$ be the relatively minimal model of $f$ and let $\mu: X \rightarrow X^{\prime}$ be its birational morphism. Assume that $q(X)=g(C)+1, L$ is $k$-very ample with $k \geq 0$, and $f^{\prime}$ has a multiple fiber. Then $K_{X} L \geq 2(k+2)(2 q(X)-4)+2(k+2)$.

Proof. By assumption $f^{\prime}$ has at least 2 multiple fibers (see Proposition 1.3 in [Se]). Let $m_{i} F_{i}$ be a multiple fiber of $f^{\prime}$. Then $F_{i}$ is a smooth elliptic curve because $q\left(X^{\prime}\right)=g(C)+1$. Then $L^{\prime}\left(m_{i}-1\right) F_{i}=L \mu^{*}\left(\left(m_{i}-1\right) F_{i}\right) \geq k+2$ by Lemma 1.9 , where $L^{\prime}=\mu_{*}(L)$ in the sense of cycle theory. Hence by the canonical bundle formula and Lemma 1.11 we get $K_{X} L \geq K_{X^{\prime}} L^{\prime} \geq$ $2(k+2)(2 q(X)-4)+2(k+2)$.

Proposition 1.13. Let $X$ be a smooth projective surface of general type. Assume that $X$ is minimal and $q(X) \geq 1$. Then $K_{X}^{2} \geq 2 p_{g} \geq 2 q(X)$. 
Proof. See Théorém 6.1 in [De].

DenITION 1.14. Let $X$ be a smooth projective surface, let $C$ be a smooth projective curve, and let $f: X \rightarrow C$ be a surjective morphism with connected fibers. Then $(f, X, C)$ is called a fiber space with $\operatorname{dim} X=2$. If $L$ is an ample line bundle, then we call $(f, X, C, L)$ a polarized fiber space.

LEMMA 1.15. Let $(f, X, C, L)$ be a polarized fiber space with $\operatorname{dim} X=2$ and $g(F) \geq 2$ for a general fiber $F$ of $f$. Then

(1) If $f$ is relatively minimal, then $K_{X / C}$ is nef, where $K_{X / C}:=K_{X}-f^{*}\left(K_{C}\right)$ is the relative canonical divisor. Furthermore if $K_{X / C}^{2}=0$ then $(f, X, C)$ is locally trivial.

(2) $K_{X / C} L \geq 0$.

(3) $K_{X / C}+L$ is nef if $\kappa(X) \geq 0$.

Proof. (1) See [Bea].

(2) See Claim 5.6 in [Fk1].

(3) See Lemma 2.5 in [Fk3].

LEMMA 1.16. Let $(f, X, C)$ be a fiber space with $\operatorname{dim} X=2$. Then $q(X) \leq g(F)+g(C)$, where $F$ is a general fiber of $f$. If $g(F) \geq 2$ and $q(X)=$ $g(F)+g(C)$, then $X \sim_{\text {bir }} F \times C$.

Proof. See Lemme in [Bea].

LEMMA 1.17. Let $(f, X, C, L)$ be a polarized fiber space with $\operatorname{dim} X=2$ and $\kappa(X)=2$. Assume that $f$ is locally trivial and $L$ is $k$-very ample with $k \geq 0$. Then $g(L) \geq(k+2) q(X)$.

Proof. By assumption there exist a smooth projective surface $S$, a smooth projective curve $B$, etale coverings $\pi: S \rightarrow X$ and $\varepsilon: B \rightarrow C$, and a fiber space $p: S \rightarrow B$ such that $S \cong B \times F, p$ is the first projective, and $\varepsilon \circ p=f \circ \pi$. By Lemma 1.12 in $[\mathrm{Fk} 1]$, we get

$$
q(X) \leq g(C)+\frac{1}{\operatorname{deg} \pi}(g(F)-1)+1
$$

for a fiber $F$ of $f$. Since $\kappa(X)=2$, we remark that $g(F) \geq 2$ and $g(C) \geq 2$. We calculate $K_{X / C} L$;

$$
\begin{aligned}
K_{X / C} L & =\left(K_{B \times F / B} \pi^{*}(L)\right) \times \frac{1}{\operatorname{deg} \pi} \\
& =(2 g(F)-2)\left(\pi^{*}(L) B\right) \times \frac{1}{\operatorname{deg} \pi} .
\end{aligned}
$$


(I) The case in which $k=0$.

Since $\pi^{*}(L) B=L \pi_{*}(B) \geq 2$ by Lemma 1.9 , we get

$$
\begin{aligned}
g(L) & =g(C)+\frac{1}{2}\left(K_{X / C}+L\right) L+(L F-1)(g(C)-1) \\
& \geq g(C)+2(g(F)-1) \times \frac{1}{\operatorname{deg} \pi}+\frac{1}{2} L^{2}+(L F-1)(g(C)-1) .
\end{aligned}
$$

If $L F \geq 3$, then

$$
\begin{aligned}
g(L) & \geq g(C)+2(g(F)-1) \times \frac{1}{\operatorname{deg} \pi}+\frac{1}{2} L^{2}+2(g(C)-1) \\
& =2\left(g(C)+\frac{1}{\operatorname{deg} \pi}(g(F)-1)+1\right)+\frac{1}{2} L^{2}+g(C)-4
\end{aligned}
$$

Then $g(L) \geq 2 q(X)$ if $L^{2} \geq 3$ because $g(C) \geq 2$.

If $L^{2}=2$, then by Proposition 1.3 we get $q(X)=0$ and $g(L)>2 q(X)$.

If $L F=2$, then

$$
\begin{aligned}
g(L) & \geq g(C)+2(g(F)-1) \times \frac{1}{\operatorname{deg} \pi}+\frac{1}{2} L^{2}+(g(C)-1) \\
& =2\left(g(C)+\frac{1}{\operatorname{deg} \pi}(g(F)-1)+1\right)+\frac{1}{2} L^{2}-3
\end{aligned}
$$

Hence $g(L) \geq 2 q(X)$ if $L^{2} \geq 5$. So we may assume that $L^{2} \leq 4$. Since Bs $\left|L_{F}\right|=\emptyset$ for any fiber of $F$, the natural map

$$
f^{*} \circ f_{*} \mathcal{O}(L) \rightarrow \mathcal{O}(L)
$$

is surjective. We put $\mathscr{E}:=f_{*} \mathcal{O}(L)$. Then since $\mathrm{Bs}\left|L_{F}\right|=\emptyset, g(F) \geq 2$, and $L F=2$, we obtain that $\mathscr{E}$ is a locally free sheaf of rank two on $C$ and there exists a double covering $\rho: X \rightarrow \boldsymbol{P}(\mathscr{E})$ such that $f=p \circ \rho$, where $\boldsymbol{P}(\mathscr{E})$ is the projective bundle of $\mathscr{E}$ on $C$ and $p: \boldsymbol{P}(\mathscr{E}) \rightarrow C$ is the bundle map. Let $B$ be the branch locus of $\rho$. Then there exists $Z \in \operatorname{Pic}(\boldsymbol{P}(\mathscr{E}))$ such that $B \in|2 Z|$. Then $K_{X}=$ $\rho^{*}\left(K_{P(\mathscr{E})}+Z\right)$. (See e.g. [Pe].) By construction $L=\rho^{*}(H(\mathscr{E}))$, where $H(\mathscr{E})$ is the tautological line bundle of $\boldsymbol{P}(\mathscr{E})$. Then $H(\mathscr{E})$ is ample and $H(\mathscr{E})^{2} \leq 2$ because $L^{2} \leq 4$. On the other hand, $h^{0}(L)=h^{0}(H(\mathscr{E}))+h^{0}(H(\mathscr{E})-Z)$. Since $K_{X} F>0$, we get that $Z F_{p}>2$ for a fiber $F_{p}$ of $p$. Hence $h^{0}(H(\mathscr{E})-Z)=0$ because $(H(\mathscr{E})-Z) F_{p}<0$. So we get $h^{0}(L)=h^{0}(H(\mathscr{E}))$ and $H(\mathscr{E})$ is spanned. But by Proposition 1.3 (2), we get that $g(C)=q(\boldsymbol{P}(\mathscr{E}))=0$. This is a contradiction.

(II) The case in which $k \geq 1$.

Since $L F \geq k+3$ and $\pi^{*}(L) B=L \pi_{*}(B) \geq k+3$ by Lemma 1.9, we get 


$$
\begin{aligned}
g(L) & =g(C)+\frac{1}{2}\left(K_{X / C}+L\right) L+(L F-1)(g(C)-1) \\
& \geq g(C)+(k+3)(g(F)-1) \times \frac{1}{\operatorname{deg} \pi}+\frac{1}{2} L^{2}+(k+2)(g(C)-1) \\
& >(k+2)\left(g(C)+\frac{1}{\operatorname{deg} \pi}(g(F)-1)+1\right)+\frac{1}{2} L^{2}-2 k-2+(g(C)-2) .
\end{aligned}
$$

If $k \geq 2$, then $L^{2} \geq 4 k+5$ by Theorem 1.7. Hence $g(L)>(k+2) q(X)$.

If $k=1$ and $L^{2} \geq 6$, then $g(L) \geq 3 q(X)$ is obtained.

If $k=1, L^{2} \leq 5$, and $h^{0}(L) \geq 5$, then $L^{2} \geq 2 \Delta(L)+1$. Since $\kappa(X)=2$ and $L$ is very ample, we get $L^{2} \geq 2$ and $g(L) \geq 3$. Hence $g(L) \geq 3>\Delta(L)$. By Theorem 1.2, this is impossible.

If $k=1, L^{2} \leq 5$, and $h^{0}(L)=4$, then $X$ is a hypersurface in $P^{3}$. So we get that $q(X)=0$ and $g(L)>3 q(X)$.

Lemma 1.18. Let $(X, L)$ be a polarized surface with $\kappa(X)=2$. Assume that $L$ is $k$-very ample with $k \geq 0$ and $X \sim_{\mathrm{bir}} F \times C$, where $\sim_{\mathrm{brr}}$ denotes birational equivalence, and $F$ and $C$ are smooth projective curves with $g(F) \geq 2$ and $g(C) \geq 2$. Then $g(L) \geq(k+2) q(X)$.

Proof. Let $\mu: X \rightarrow F \times C$ be the minimalization of $X$ and $p: F \times C \rightarrow C$ the second projection. Let $f:=p \circ \mu$. Then $K_{X} \equiv \mu^{*}((2 g(F)-2) C+(2 g(C)-2) F)$ $+E_{\mu}$, where $E_{\mu}$ is a $\mu$-exceptional effective divisor. So we get

$$
\begin{aligned}
K_{X} L & \geq(2 g(F)-2) L \mu^{*}(C)+(2 g(C)-2) L \mu^{*}(F) \\
& \geq(k+2)(2 g(F)+2 g(C)-4)
\end{aligned}
$$

by Lemma 1.9 .

(I) The case in which $k \geq 2$.

By Theorem 1.7, we get $L^{2} \geq 4 k+5$. Hence

$$
\begin{aligned}
g(L) & \geq 1+(k+2)(g(F)+g(C)-2)+2 k+\frac{5}{2} \\
& =(k+2) q(X)-\frac{1}{2} .
\end{aligned}
$$

So we obtain $g(L) \geq(k+2) q(X)$.

(II) The case in which $k=1$.

If $L^{2} \geq 9$, then by the same argument as in the case (I) we get $g(L) \geq 3 q(X)$. So we may assume that $L^{2} \leq 8$. We remark that $L^{2} \geq 2$ since $\kappa(X)=2$. Hence $g(L) \geq 3$.

If $h^{0}(L) \geq 6$ and $L^{2} \leq 7$, then $L^{2} \geq 2 \Delta(L)+1$ and $g(L) \geq 3 \geq \Delta(L)$. Hence by Theorem 1.2 this is impossible.

If $h^{0}(L) \geq 6$ and $L^{2}=8$, then $\Delta(L) \leq 4$. Since $L^{2}=8$ we get $g(L) \geq$ $6>\Delta(L)$. If $\Delta(L)=4$, then by Theorem 1.2 we get $q(X)=0$. But this is 
a contradiction because $q(X)=g(F)+g(C) \geq 4$. If $\Delta(L) \leq 3$, then $L^{2} \geq$ $2 \Delta(L)+1$ and this is impossible by Theorem 1.2.

If $h^{0}(L)=5$ and $L^{2} \leq 5$, then $g(L)>2 \geq \Delta(L)$ and $L^{2} \geq 2 \Delta(L)+1$. But by Theorem 1.2, this is impossible.

If $h^{0}(L)=5$ and $L^{2}=6$, then $g(L) \geq 5>3=\Delta(L)$ and $L^{2}=2 \Delta(L)$. Hence by Theorem 1.2 we get $q(X)=0$. But this is impossible because $q(X)=$ $g(C)+g(F) \geq 4$.

If $h^{0}(L)=5$ and $L^{2}=7$, then by Proposition 1.6 we get

$$
10(g(L)-1)=14-4(g(F)-1)(g(C)-1)+2 a,
$$

where $a:=8(g(F)-1)(g(C)-1)-K_{X}^{2}$.

We remark that $a$ is nonnegative integer and $K_{X} L \geq 3(2 g(F)+2 g(C)-4)+a$. Since $g(F) \geq 2$ and $g(C) \geq 2$, we get that $14-4(g(F)-1)(g(C)-1) \leq 10$. So we get $a \geq 15$ because $g(L) \geq 5$. Hence $K_{X} L \geq 3(2 q(X)-4)+15$ and we get $g(L)>3 q(X)$.

If $h^{0}(L)=5$ and $L^{2}=8$, then by Proposition 1.6 we get

$$
10(g(L)-1)=24-4(g(F)-1)(g(C)-1)+2 a .
$$

(We use the same notation as above.) Since $g(F) \geq 2$ and $g(C) \geq 2$, we get that $24-4(g(F)-1)(g(C)-1) \leq 20$. So we get $a \geq 15$ because $g(L) \geq 6$. Hence $K_{X} L \geq 3(2 q(X)-4)+15$ and we get $g(L)>3 q(X)$.

(III) The case in which $k=0$.

If $L^{2} \geq 5$, then by the same argument as in the case (I) we get $g(L) \geq 2 q(X)$. So we may assume $L^{2} \leq 4$.

(III-1) The case in which $L^{2}=4$.

If $X$ is not minimal, then

$$
\begin{aligned}
K_{X} L & \geq 4(g(F)+g(C)-2)+1 \\
& =4 q(X)-7
\end{aligned}
$$

Hence we get $g(L) \geq 2 q(X)$. So we may assume that $X$ is minimal. But then by Lemma 1.17 we get $g(L) \geq 2 q(X)$.

(III-2) The case in which $L^{2}=3$.

By the same argument as in the case (I) we get $g(L) \geq 2 q(X)-1$. Assume that $g(L)=2 q(X)-1$. Then $K_{X} L=4 q(X)-7$. In particular, $\mu$ is a simple blowing up of $F \times C$, and $L F=2$ for a general fiber $F$ of $f$. Let $F_{e}:=F_{1}+E$ be a fiber of $f$, where $F_{1}$ is a smooth curve of genus $g\left(F_{1}\right) \geq 2$ and $E$ is the (-1)curve of $\mu$. Since $L$ is ample and $L F=2$, we get $L F_{1}=L E=1$. But this is impossible because Bs $|L|=\emptyset$ and $g\left(F_{1}\right) \neq 0$.

(III-3) The case in which $L^{2} \leq 2$.

Then by Proposition $1.3(2)$, we get $q(X)=0$ and this is a contradiction because $q(X)=g(F)+g(C) \geq 4$.

This completes the proof of Lemma 1.18. 
Proposition 1.19 (Castelnuovo's bound). Let $(X, L)$ be a polarized surface. Assume that $L$ is very ample with $N=h^{0}(L)-2$ and $d=L^{2}$. Then

$$
g(L) \leq\left\lfloor\frac{d-2}{N-1}\right\rfloor\left(d-N-\left(\left\lfloor\frac{d-2}{N-2}\right\rfloor-1\right) \frac{N-1}{2}\right) .
$$

Proof. See [ACGH].

\section{§2. The case in which $\kappa(X)=0$}

THEOREM 2.1. Let $(X, L)$ be a polarized surface with $\kappa(X)=0$. Assume that $L$ is $k$-very ample with $k \geq 0$. Then $g(L) \geq(k+2) q(X)$. Furthermore if $g(L)=(k+2) q(X)$, then $(X, L)$ is one of the following;

(1) $(X, L)$ is a polarized abelian surface with $L^{2}=4 k+6$,

(2) $k=0, X$ is a one point blowing up of $S$, and $L=\mu^{*}(A)-2 E$, where $S$ is an abelian surface, $A$ is an ample line bundle with $A^{2}=8, \mu: X \rightarrow S$ is its blowing $u p$, and $E$ is a (-1)-curve of $\mu$.

Proof. (I) The case in which $k=0$.

(I-A) The proof of $g(L) \geq 2 q(X)$.

By the classification theory of surfaces, we get $q(X) \leq 2$.

If $q(X) \leq 1$, then $g(L) \geq 2 \geq 2 q(X)$.

If $q(X)=2$ and $g(L) \geq 4$, then $g(L) \geq 2 q(X)$.

If $q(X)=2$ and $g(L) \leq 3$, then $L^{2} \leq 4$. If $g(L) \leq 2$, then $L^{2} \leq 2$ and by Proposition 1.3 (2) we get $q(X)=0$ and this is impossible. If $g(L)=3$ and $L^{2}=4$, then $X$ is an abelian surface. But then $h^{0}(L)=2$ and this is impossible. If $g(L)=3$ and $L^{2} \leq 2$, then by Proposition $1.3(2)$ we get $q(X)=0$ and this is a contradiction. If $g(L)=3, L^{2}=3$, and $h^{0}(L) \geq 4$, then $L^{2} \geq 2 \Delta(L)+1$ and $g(L)>\Delta(L)$. But by Theorem 1.2 this is impossible. If $g(L)=3, L^{2}=3$, and $h^{0}(L)=3$, then there exists a triple covering $\varphi_{|L|}: X \rightarrow \boldsymbol{P}^{2}$ defined by $|L|$. Since $K_{X} L=1$, we get that $K_{X}^{2}=-1$. But by Proposition 1.4 , this is impossible because $\chi\left(\mathcal{O}_{X}\right)=0$.

Therefore we get $g(L) \geq 2 q(X)$.

(I-B) The classification of $(X, L)$ with $g(L)=2 q(X)$.

First we assume that $q(X) \leq 1$. Since $\kappa(X)=0$, we get $q(X)=1$ and $g(L)=2$. But by Lemma 1.5 this is impossible. So we assume that $q(X)=2$. Then $g(L)=2 q(X)=4$ and $L^{2} \leq 6$.

(I-B-1) The case in which $L^{2} \leq 2$.

Then by Proposition 1.3 (2) this is impossible.

(I-B-2) The case in which $L^{2}=3$.

If $h^{0}(L) \geq 4$, then $\Delta(L) \leq 1$. By Theorem $1.2(1)$, we get that $\Delta(L)=1$ because $\kappa(X)=0$. Then $L^{2}>2 \Delta(L)$ and $g(L)>\Delta(L)$. Hence this is impossible by Theorem 1.2. So we may assume that $h^{0}(L)=3$. Then there exists a 
triple covering $\pi: X \rightarrow \boldsymbol{P}^{2}$ defined by $|L|$. Here we use Proposition 1.4. Since $\chi\left(\mathcal{O}_{X}\right)=0$ and $g(L)=4$, we get $c_{2}=12$. On the other hand,

$$
\begin{aligned}
K_{X}^{2} & =2 g(L)^{2}-4 g(L)+11-3 c_{2} \\
& =-9 .
\end{aligned}
$$

But since $K_{X} L=3$, this is a contradiction.

(I-B-3) The case in which $L^{2}=4$.

In this case $K_{X} L=2$. In particular $X$ is not minimal. Let $\mu: X \rightarrow S$ be the minimalization of $X$. Then $S$ is an abelian surface. Let $A:=\mu_{*}(L)$. Then $A^{2}=8$ or 6 .

(I-B-3-1) The case in which $A^{2}=6$.

Then $\mu$ is a composition of two blowing ups. By Lemma 1.8 (2) we get Bs $|A|=\emptyset$. But since $h^{0}(A)=3$ and $h^{0}(L)<h^{0}(A)$, this is impossible.

(I-B-3-2) The case in which $A^{2}=8$.

Then $\mu$ is one point blowing up and $L=\mu^{*}(A)-2 E$, where $E$ is a (-1)curve of $\mu$. This is the type (2) is Theorem 2.1 .

(I-B-4) The case in which $L^{2}=5$.

In this case, $K_{X} L=1$. Then $L=\mu^{*}(A)-E$ and $A^{2}=6$ and $h^{0}(A)=3$. By Lemma 1.8 (2) we get Bs $|A|=\emptyset$. But then $h^{0}(L)<h^{0}(A)=3$ and this is impossible.

(I-B-5) The case in which $L^{2}=6$.

Then this is the type (1) in Theorem 2.1 .

(II) The case in which $k=1$.

(II-A) The proof of $g(L) \geq 3 q(X)$.

If $q(X) \leq 1$ and $g(L) \geq 3$, then $g(L) \geq 3 q(X)$.

If $q(X) \leq 1$ and $g(L) \leq 2$, then $L^{2} \leq 2$. Since $L$ is very ample with $\kappa(X)=0$, we get that $h^{0}(L) \geq 4$. But then $\Delta(L) \leq 0$ and $\kappa(X)=-\infty$, a contradiction.

If $q(X)=2$ and $g(L) \geq 6$, then $g(L) \geq 3 q(X)$.

If $q(X)=2$ and $g(L) \leq 5$, then $L^{2} \leq 8$. We remark that $h^{0}(L) \geq 5$ in this case. (By the above we get $h^{0}(L) \geq 4$. If $h^{0}(L)=4$, then $X$ is a hypersurface of $\boldsymbol{P}^{3}$. But then $q(X)=0$ and this is a contradiction.) Hence $\Delta(L) \leq L^{2}-3$.

(II-A-1) The case in which $L^{2} \leq 5$.

Then $L^{2} \geq 2 \Delta(L)+1$ and $g(L) \geq 2 \geq \Delta(L)$. But by Theorem 1.2 this is impossible because $\kappa(X)=0$.

(II-A-2) The case in which $L^{2}=8$.

Then $X$ is an abelian surface and $h^{0}(L)=4$. But this is impossible because $h^{0}(L) \geq 5$.

(II-A-3) The case in which $L^{2}=7$.

Then $X$ is not minimal. Let $\mu: X \rightarrow S$ be the minimalization of $X$. Then $S$ is an abelian surface. Let $A:=\mu_{*}(L)$. Then $L=\mu^{*}(A)-E, A^{2}=8$, and $h^{0}(A)=4$ since $g(L) \leq 5$. By Lemma 1.8 (2) we get $\mathrm{Bs}|A|=\emptyset$. But then $h^{0}(L)<h^{0}(A)=4$ and this is impossible.

(II-A-4) The case in which $L^{2}=6$. 
Then $X$ is not minimal. Let $\mu: X \rightarrow S$ be the minimalization of $X$. Then $S$ is an abelian surface. Let $A:=\mu_{*}(L)$. Then $A^{2}=10$ or 8 because $K_{X} L \leq 2$. (II-A-4-1) The case in which $A^{2}=8$.

Then $\mu$ is a composition of two simple blowing ups. By Lemma 1.8 (2) we get Bs $|A|=\emptyset$. But $h^{0}(A)=4$ and $h^{0}(L)<h^{0}(A)$, this is impossible.

(II-A-4-2) The case in which $A^{2}=10$.

Then $\mu$ is one point blowing up and $L=\mu^{*}(A)-2 E$, where $E$ is a $(-1)$-curve of $\mu$. By Lemma 1.8 (2) we get Bs $|A|=\emptyset$. But $h^{0}(A)=5$ and $h^{0}(L)<h^{0}(A)$, so this is impossible.

Therefore $g(L) \geq 3 q(X)$.

(II-B) The classification of $(X, L)$ with $g(L)=3 q(X)$.

Since $q(X) \leq 2$, we get two possibilities; $(g(L), q(X))=(3,1),(6,2)$.

(II-B-1) The case in which $(g(L), q(X))=(3,1)$.

Then $L^{2} \leq 4$. Since $q(X)=1$, we get that $h^{0}(L) \geq 5$ by the same argument as above. Hence $\Delta(L) \leq 1$. By Theorem 1.2 we get that $\Delta(L)=1$ and $L^{2}=4$. Hence $X$ is minimal and $h^{0}(L)=L^{2} / 2=2$. This is impossible.

(II-B-2) The case in which $(g(L), q(X))=(6,2)$.

Assume that $L^{2} \leq 9$. Then $X$ is not minimal. Let $\mu: X \rightarrow S$ be the minimalization of $X$. Then $S$ is an abelian surface. Let $A:=\mu_{*}(L)$. We remark that $h^{0}(L) \geq 5$.

(II-B-2-1) The case in which $L^{2} \leq 5$.

Then $L^{2} \geq 2 \Delta(L)+1$ and $g(L)>\Delta(L)$. But this is impossible by Theorem 1.2.

(II-B-2-2) The case in which $L^{2} \geq 6$.

Then $K_{X} L \leq 4$. If $h^{0}(L)=5$, then by Proposition 1.6 this is impossible. (We remark that the value of $K_{X}^{2}$ is $-1,-2,-3$, or -4 .) So we may assume that $h^{0}(L) \geq 6$.

(II-B-2-2-a) The case in which $6 \leq L^{2} \leq 7$.

Then $L^{2} \geq 2 \Delta(L)+1$ and $g(L) \geq \Delta(L)$. But this is impossible by Theorem 1.2.

(II-B-2-2-b) The case in which $L^{2}=8$.

Then $A^{2}=12$ or 10 .

(b-1) The case in which $A^{2}=10$.

Then $\mu$ is a composition of two simple blowing ups. By Lemma 1.8 (2) we get Bs $|A|=\emptyset$. But $h^{0}(A)=5$ and $h^{0}(L)<h^{0}(A)$, this is impossible.

(b-2) The case in which $A^{2}=12$.

Then $\mu$ is one point blowing up and $L=\mu^{*}(A)-2 E$, where $E$ is a (-1)-curve of $\mu$. By Lemma $1.8(2)$ we get Bs $|A|=\emptyset$. But $h^{0}(A)=6$ and $h^{0}(L)<h^{0}(A)$, this is impossible.

(II-B-2-2-c) The case in which $L^{2}=9$.

Then $L=\mu^{*}(A)-E$ and $A^{2}=10$ and $h^{0}(A)=5$. By Lemma 1.8 (2) we get Bs $|A|=\emptyset$. But then $h^{0}(L)<h^{0}(A)=5$ and this is impossible.

Therefore $L^{2}=10$. In this case $X$ is an abelian surface. This is the type (1) in Theorem 2.1.

(III) The case in which $k \geq 2$.

(III-A) The proof of $g(L) \geq(k+2) q(X)$.

If $q(X)=0$, then $g(L)>(k+2) q(X)$. 
If $q(X)=1$ or 2 , then by Theorem 1.7 we get that $L^{2} \geq 4 k+5$. Hence

$$
\begin{aligned}
g(L) & \geq 1+\left\lceil\frac{4 k+5}{2}\right\rceil \\
& =2 k+4 \\
& \geq(k+2) q(X) .
\end{aligned}
$$

(III-B) The classification of $(X, L)$ with $g(L)=(k+2) q(X)$.

By the above argument, we get $q(X) \neq 0$ and so we get $L^{2} \geq 4 k+5$ by Theorem 1.7. Therefore there exist two possibilities; $\left(L^{2}, K_{X} L\right)=(4 k+6,0)$, $(4 k+5,1)$. If $\left(L^{2}, K_{X} L\right)=(4 k+5,1)$, then $X$ is one point blowing up of an abelian surface and $L=\mu^{*}(A)-E$, where $\mu: X \rightarrow S$ is the minimalization of $X$ and $A:=\mu_{*}(L)$. But this is impossible because $1=K_{X} L=E L \geq k \geq 2$ by Lemma 1.9.

If $\left(L^{2}, K_{X} L\right)=(4 k+6,0)$, then $X$ is an abelian surface and this is the type (1) in Theorem 2.1.

This completes the proof of Theorem 2.1.

\section{§3. The case in which $\kappa(X)=1$}

THEOREM 3.1. Let $(X, L)$ be a polarized surface such that $\kappa(X)=1$. Assume that $L$ is $k$-very ample with $k \geq 0$. Then $g(L) \geq(k+2) q(X)$. Furthermore if $g(L)=(k+2) q(X)$, then $(X, L)$ is one of the following:

(1) $k=0, L^{2}=4, q(X)=3, X$ has a locally trivial elliptic fibration $f: X \rightarrow C$, and $L F=3$ for a fiber $F$ of $f$, where $C$ is a smooth projective curve with $g(C)=2$.

(2) $k \geq 1, L^{2}=4 k+6, q(X) \geq 3, X$ has a locally trivial elliptic fibration $f: X \rightarrow C$, and $L F=k+2$ for a fiber $F$ of $f$, where $C$ is a smooth projective curve with $g(C)=q(X)-1$.

Proof. Since $\kappa(X)=1$, there exists an elliptic fibration $f: X \rightarrow C$, where $C$ is a smooth projective curve. Then we remark that $q(X)=g(C)$ or $q(X)=g(C)+1$.

(I) The case in which $k=0$.

ClaIM 3.2. $L^{2} \geq 2$. If $L^{2}=2$, then $q(X)=0$ and $g(L)>2 q(X)$.

Proof. If $L^{2}=1$, then $\Delta(L)=0$ and by Theorem 1.2 this is impossible. If $L^{2}=2$, then by Proposition 1.3 we get $q(X)=0$. In particular $g(L)>$ $2 q(X)$. 
(I-1) The case in which $q(X)=g(C)$.

Then $K_{X} L \geq(2 q(X)-2) L F$ by the canonical bundle formula, where $F$ is a fiber of $f$.

If $q(X)=0$, then $g(L)>2 q(X)$.

If $q(X) \geq 1$, then $K_{X} L \geq(2 q(X)-2) L F \geq 4(q(X)-1)$ by Lemma 1.9. So we obtain $g(L) \geq 2 q(X)-1+(1 / 2) L^{2}$. Since $g(L) \in Z$, we get that $g(L)>2 q(X)$ by Claim 3.2 .

(I-2) The case in which $q(X)=g(C)+1$.

(I-2-A) The proof of $g(L) \geq 2 q(X)$.

Assume that $q(X) \leq 1$. By Claim 3.2, we get $g(L) \geq 3>2 q(X)$.

Next we assume that $q(X) \geq 2$. By the canonical bundle formula and Lemma 1.9 , we get $K_{X} L \geq(2 q(X)-4) L F \geq 4(q(X)-2)$. Hence $g(L) \geq$ $2 q(X)-3+(1 / 2) L^{2}$. If $L^{2} \geq 5$, then $g(L) \geq 2 q(X)$. So we may assume that $L^{2}=3$ or 4 by Claim 3.2 .

(I-2-A-1) The case in which $L^{2}=4$.

Assume that $g(L)<2 q(X)$. Then if $X$ is not minimal or minimal such that $f$ has a multiple fiber, then by Lemma 1.9 we get $K_{X} L \geq(2 q(X)-4) L F+$ $1 \geq 4 q(X)-7$ and we obtain that $g(L) \geq 2 q(X)$. So we may assume that $X$ is minimal and $f$ has no multiple fiber. In particular any fiber of $f$ is smooth because $q(X)=g(C)+1$. Then $K_{X} \equiv(2 q(X)-4) F$. Since $\kappa(X)=1$, we get that $q(X) \geq 3$. By assumption and Lemma 1.9, we get that $L F=2$. Since $L F=2$ and any fiber of $f$ is smooth, the natural map

$$
f^{*} \circ f_{*} \mathcal{O}(L) \rightarrow \mathcal{O}(L)
$$

is surjective. We put $\mathscr{E}:=f_{*} \mathcal{O}(L)$. Then $\mathscr{E}$ is a locally free sheaf of rank two on $C$ and there exists a double covering $\pi: X \rightarrow \boldsymbol{P}(\mathscr{E})$ such that $f=p \circ \pi$, where $\boldsymbol{P}(\mathscr{E})$ is the projective bundle of $\mathscr{E}$ on $C$ and $p: \boldsymbol{P}(\mathscr{E}) \rightarrow C$ is the bundle map. Let $B$ be the branch locus of $\pi$. Then there exists $Z \in \operatorname{Pic}(\boldsymbol{P}(\mathscr{E}))$ such that $B \in|2 Z|$. Then $K_{X}=\pi^{*}\left(K_{P(\mathscr{E})}+Z\right)$. (See e.g. [Pe].) By construction $L=$ $\pi^{*}(H(\mathscr{E}))$, where $H(\mathscr{E})$ is the tautological line bundle of $\boldsymbol{P}(\mathscr{E})$. Then $H(\mathscr{E})$ is ample and $H(\mathscr{E})^{2}=2$ because $L^{2}=4$. On the other hand, $h^{0}(L)=h^{0}(H(\mathscr{E}))+$ $h^{0}(H(\mathscr{E})-Z)$. Since $K_{X} F=0$, we get that $Z F_{p}=2$ for a fiber $F_{p}$ of $p$. Hence $h^{0}(H(\mathscr{E})-Z)=0$ because $(H(\mathscr{E})-Z) F_{p}<0$. So we get $h^{0}(L)=h^{0}(H(\mathscr{E}))$ and $H(\mathscr{E})$ is spanned. But by Proposition 1.3, we get that $g(C)=q(\boldsymbol{P}(\mathscr{E}))=0$. Hence $q(X)=g(C)+1=1$ and this is a contradiction.

(I-2-A-2) The case in which $L^{2}=3$.

Assume that $g(L)<2 q(X)$. If the relatively minimal model of $f$ has a multiple fiber, then by Lemma 1.12 we get $K_{X} L \geq 4(2 q(X)-4)+4$ and we can prove $g(L) \geq 2 q(X)$ since $q(X) \geq 2$. So we may assume that the relatively minimal model of $f$ has no multiple fiber. Then $q(X) \geq 3$ since $\kappa(X)=1$. Since $L^{2}=3$, we get $K_{X} L$ is odd. Hence $X$ is not minimal. By Lemma 1.10 , we get $L F \geq 3$. But then $K_{X} L \geq 6(q(X)-2)+1$. So we get $g(L) \geq 2 q(X)$ and this is a contradiction.

(I-B) The classification of $(X, L)$ with $g(L)=2 q(X)$. 
By the above proof, we get $q(X)=g(C)+1$. First we study the upper bound of $L^{2}$. Since $K_{X} L \geq 2(q(X)-2) L F \geq 4(q(X)-2)$, we get $g(L) \geq 2 q(X)-3+$ $(1 / 2) L^{2}$. Hence $L^{2} \leq 6$.

(I-B-1) The case in which $L^{2}=6$.

Then $X$ is minimal, $L F=2$, and $K_{X} \equiv(2 q(X)-4) F$. In particular any fiber of $f$ is smooth and $q(X) \geq 3$. Since $L F=2$ and any fiber of $f$ is smooth, the natural map

$$
f^{*} \circ f_{*} \mathcal{O}(L) \rightarrow \mathcal{O}(L)
$$

is surjective. We put $\mathscr{E}:=f_{*} \mathcal{O}(L)$. Then $\mathscr{E}$ is a locally free sheaf of rank two on $C$ and there exists a double covering $\pi: X \rightarrow \boldsymbol{P}(\mathscr{E})$ such that $f=p \circ \pi$, where $\boldsymbol{P}(\mathscr{E})$ is the projective bundle of $\mathscr{E}$ on $C$ and $p: \boldsymbol{P}(\mathscr{E}) \rightarrow C$ is the bundle map. By construction $L=\pi^{*}(H(\mathscr{E}))$, where $H(\mathscr{E})$ is the tautological line bundle of $\boldsymbol{P}(\mathscr{E})$. Then $H(\mathscr{E})$ is ample and $H(\mathscr{E})^{2}=3$ because $L^{2}=6$. By the same argument as above, we get $h^{0}(L)=h^{0}(H(\mathscr{E}))$ and $H(\mathscr{E})$ is spanned.

If $h^{0}(H(\mathscr{E})) \geq 4$, then $\Delta(H(\mathscr{E})) \leq 1$. If $g(H(\mathscr{E}))=0$, then $q(\boldsymbol{P}(\mathscr{E}))=0$ and this is a contradiction. If $g(H(\mathscr{E})) \geq 1$, then $g(H(\mathscr{E})) \geq \Delta(H(\mathscr{E}))$ and $H(\mathscr{E})^{2} \geq$ $2 \Delta(H(\mathscr{E}))+1$. But by Theorem $1.2 q(\boldsymbol{P}(\mathscr{E}))=0$ and this is impossible.

If $h^{0}(H(\mathscr{E}))=3$, then there exists a triple cover $\varphi_{|H(\mathscr{E})|}: \boldsymbol{P}(\mathscr{E}) \rightarrow \boldsymbol{P}^{2}$ defined by $H(\mathscr{E})$. Since $g(H(\mathscr{E}))=g(C), K_{P(\mathscr{E})}^{2}=8(1-g(C))$ and $\chi\left(\mathcal{O}_{\boldsymbol{P}(\mathscr{E})}\right)=1-g(C)$, we get that $g(C)=0$ or 1 by Proposition 1.4. But this is a contradiction because $3 \leq q(X)=g(C)+1$.

(I-B-2) The case in which $L^{2}=5$.

Since $L^{2}=5$, we obtain $g(L) \geq 4$. Hence $q(X) \geq 2$ because $g(L)=2 q(X)$. Assume that the relatively minimal model of $f$ has a multiple fiber. Then by Lemma 1.12, we get that $g(L)>2 q(X)$ and this is a contradiction. Hence the relatively minimal model of $f$ has no multiple fiber. This fact induces $q(X) \geq 3$. Since $L^{2}$ is odd, we get that $X$ is not relatively minimal by the canonical bundle formula. By Lemma 1.10 , we get $L F \geq 3$ for a fiber $F$ of $f$. Hence $K_{X} L \geq$ $3(2 q(X)-4)+1$ and we get $g(L) \geq 2 q(X)+q(X)-2>2 q(X)$. This is a contradiction.

(I-B-3) The case in which $L^{2}=4$.

By the same argument as in the case (I-B-2), the relatively minimal model of $f$ has no multiple fiber. In particular $q(X) \geq 3$. By Lemma 1.9 (2), we get $L F \geq 2$ for a general fiber $F$. If $L F=2$, then $f$ is relatively minimal by Lemma 1.10. Because $K_{X} \equiv(2 q(X)-4) F$, we get $K_{X} L=4 q(X)-8$. Since $L^{2}=4$, we get $g(L)=2 q(X)-1$. But this is a contradiction because $g(L)=2 q(X)$. So we get $L F \geq 3$. Hence $K_{X} L \geq 3(2 q(X)-4)$ and we get $g(L) \geq 2 q(X)+q(X)-3$. Since $g(L)=2 q(X)$, we get that $f$ is relatively minimal and $q(X)=3$. In particular $f$ is a locally trivial fibration. This is the type (1) in Theorem 3.1.

(I-B-4) The case in which $L^{2}=3$.

By the same argument as in the case (I-B-2), the relatively minimal model of $f$ has no multiple fiber. In particular $q(X) \geq 3$. Furthermore $X$ is not minimal because $L^{2}$ is odd. 
If $h^{0}(L) \geq 4$, then $\Delta(L) \leq 1$. Since $L^{2}=3$, we obtain $g(L) \geq 3$. Hence $g(L)>\Delta(L)$ and $L^{2} \geq 2 \Delta(L)+1$. But by Theorem 1.2 this is impossible.

So we assume that $h^{0}(L)=3$. Then there exists a triple covering $\varphi_{|L|}: X \rightarrow \boldsymbol{P}^{2}$ which is defined by $|L|$. We remark that $\chi\left(\mathcal{O}_{X}\right)=0$ and $K_{X}^{2}<0$. By Proposition 1.4, we have the following equalities:

$$
\begin{aligned}
0 & =\frac{1}{2} g(L)(g(L)+1)+2-c_{2}, \\
K_{X}^{2} & =2 g(L)^{2}-4 g(L)+11-3 c_{2} .
\end{aligned}
$$

By these equalities we have $2 K_{X}^{2}=(g(L)-1)(g(L)-10)$. Since $K_{X}^{2}<0$, we get that $1<g(L)<10$. By assumption $g(L)$ is even. Hence $g(L)=2,4,6,8$. By the above, we get $K_{X}^{2}=-4$ (resp. $\left.-9,-10,-7\right)$ if $g(L)=2$ (resp. 4, 6, 8). In particular, $X$ is at least four times blowing up of the relatively minimal model of $f$. By using Lemma 1.10 and the canonical bundle formula, we get $K_{X} L \geq$ $3(2 q(X)-4)+4$ and we obtain $g(L) \geq 2 q(X)+q(X)-(3 / 2)>2 q(X)$ because $q(X) \geq 3$. This is impossible.

(II) The case in which $k=1$.

ClaIm 3.3. $h^{0}(L) \geq 5, L^{2} \geq 5$, and $g(L) \geq 4$.

Proof. Since $L$ is very ample and $\kappa(X)=1$, we get that $h^{0}(L) \geq 4$. If $h^{0}(L)=4$, then there exists an embedding $X \rightarrow \boldsymbol{P}^{3}$. But since $\kappa(X)=1$, this is impossible. Therefore $h^{0}(L) \geq 5$.

If $L^{2} \leq 3$, then $\Delta(L)=0$ and this is impossible by Theorem 1.2. Hence $L^{2} \geq 4$. Since $K_{X} L>0$, we get $g(L) \geq 4$.

If $L^{2}=4$, then $\Delta(L) \leq 1$. We get $g(L)>\Delta(L)$ and $L^{2}>2 \Delta(L)+1$. But this is a contradiction by Theorem 1.2.

(II-A) The proof of $g(L) \geq 3 q(X)$.

(II-A-1) The case in which $L^{2} \geq 9$.

If $q(X) \leq 1$, then $g(L) \geq 4>3 \geq 3 q(X)$ by Claim 3.3. So we assume that $q(X) \geq 2$. Then by Lemma 1.9 and the canonical bundle formula, we get $K_{X} L \geq 3(2 q(X)-4)$ since $q(X)=g(C)$ or $q(X)=g(C)+1$. Hence $g(L) \geq$ $3 q(X)-5+(1 / 2) L^{2}$ and we get $g(L) \geq 3 q(X)$.

(II-A-2) The case in which $L^{2}=7$ or 8 .

If $h^{0}(L) \geq 6$, then $\Delta(L) \leq 4$.

If $\Delta(L)=4$, then $L^{2}=8$ and $h^{0}(L)=6$. In particular, $L^{2}=2 \Delta(L)$. On the other hand $g(L) \geq 6>\Delta(L)$. By Theorem 1.2 we get $g(L)>3 q(X)$.

If $\Delta(L) \leq 3$, then $L^{2} \geq 7 \geq 2 \Delta(L)+1$ and $g(L) \geq 5>\Delta(L)$. Hence by Theorem 1.2 this is a contradiction.

Assume that $h^{0}(L)=5$. If $q(X)=g(C)$, then $K_{X} L \geq 3(2 q(X)-2)$ and $g(L)>3 q(X)$. So we may assume $q(X)=g(C)+1$. Then $\chi\left(\mathcal{O}_{X}\right)=0$.

Assume that $L^{2}=8$. Then by Proposition 1.6 we get that $K_{X}^{2} \leq-13$ since $g(L) \geq 6$. Hence $K_{X} L \geq 3(2 q(X)-4)+13$ by the canonical bundle formula. So we get $g(L)>3 q(X)$. 
Assume that $L^{2}=7$. By Proposition 1.6, we get that $K_{X}^{2} \leq-13$ since $g(L) \geq 5$. Therefore $K_{X} L \geq 3(2 q(X)-4)+13$ by the canonical bundle formula. So we get $g(L)>3 q(X)$.

(II-A-3) The case in which $L^{2}=6$.

Then $\Delta(L) \leq 3$. Since $L^{2}=6$, we get that $L^{2} \geq 2 \Delta(L)$ and $g(L) \geq$ $5>\Delta(L)$.

If $L^{2}=2 \Delta(L)$, then by Theorem 1.2 we get $g(L)>3 q(X)$.

If $L^{2} \geq 2 \Delta(L)+1$, then this is a contradiction because $g(L)>\Delta(L)$.

(II-A-4) The case in which $L^{2}=5$.

Then $\Delta(L) \leq 2<4 \leq g(L)$. Since $L^{2} \geq 2 \Delta(L)+1$, we get $g(L)=\Delta(L)$ by Theorem 1.2. But this is a contradiction.

(II-B) The classification of $(X, L)$ with $g(L)=3 q(X)$.

By the proof of the above, we get $g(L)>3 q(X)$ if $L^{2} \leq 8$. So we get that $L^{2} \geq 9$. By Claim 3.3 and the assumption, we get $q(X) \geq 2$. Since $K_{X} L \geq$ $3(2 q(X)-4)$, we get that $g(L) \geq 3 q(X)-5+\left(L^{2}\right) / 2$. Hence $L^{2} \leq 10$.

(II-B-1) The case in which $L^{2}=10$.

Then $f$ is the relatively minimal elliptic fibration, $f$ has no multiple fiber, and $q(X)=g(C)+1$. In particular, $f$ is a locally trivial fibration. Since $\kappa(X)=1$, we get that $q(X) \geq 3$ by the canonical bundle formula. This is the type (2) in Theorem 3.1.

(II-B-2) The case in which $L^{2}=9$.

If $q(X)=g(C)$, then $K_{X} L \geq 3(2 q(X)-2)$ by Lemma 1.9 and the canonical bundle formula. Hence $g(L) \geq 3 q(X)+(5 / 2)>3 q(X)$. So we get that $q(X)=$ $g(C)+1$. If the relatively minimal model of $f$ has a multiple fiber, then by Lemma 1.12 we get $K_{X} L \geq 6(2 q(X)-4)+6$. So we have $g(L) \geq 3 q(X)+$ $3 q(X)-(7 / 2)$. Since $q(X) \geq 2$, we get that $g(L)>3 q(X)$. Hence the relatively minimal model of $f$ has no multiple fiber. In particular $q(X) \geq 3$ because $\kappa(X)=1$. Since $L^{2}$ is odd, $f$ is not relatively minimal. By Lemma 1.10 , we have $L F \geq 4$. Hence $K_{X} L \geq 4(2 q(X)-4)+1$ and we get $g(L) \geq 3 q(X)+$ $q(X)-2$. Since $q(X) \geq 3, g(L)>3 q(X)$ is obtained and this is a contradiction.

(III) The case in which $k \geq 2$.

By Theorem 1.7, we obtain $L^{2} \geq 4 k+5$. By Lemma 1.9, we get $L F \geq k+2$ for a general fiber $F$ of $f$.

(III-A) The proof of $g(L) \geq(k+2) q(X)$.

If $q(X) \leq 1$, then

$$
\begin{aligned}
g(L) & =1+\frac{1}{2}\left(K_{X}+L\right) L \\
& >1+\frac{1}{2}(4 k+5) \\
& =2 k+\frac{7}{2} \\
& >(k+2) q(X) .
\end{aligned}
$$


If $q(X) \geq 2$, then $K_{X} L \geq(k+2)(2 q(X)-4)$ by the canonical bundle formula. Hence $g(L) \geq(k+2) q(X)-(1 / 2)$. Since $g(L) \in Z$, we get $g(L) \geq(k+2) q(X)$.

(III-B) The classification of $(X, L)$ with $g(L)=(k+2) q(X)$.

By the above proof we get that $q(X)=g(C)+1$ and $q(X) \geq 2$ in this case. If the relatively minimal model of $f$ has a multiple fiber, then by Lemma 1.12 we get $K_{X} L \geq 2(k+2)(2 q(X)-4)+2(k+2)$. So we have $g(L) \geq$ $(k+2) q(X)+(k+2) q(X)-k-(5 / 2)$. Since $q(X) \geq 2$, we get that $g(L)>$ $(k+2) q(X)$. Hence the relatively minimal model of $f$ has no multiple fiber. In particular $q(X) \geq 3$ because $\kappa(X)=1$. On the other hand since

$$
(k+2) q(X)=g(L) \geq 1+\frac{1}{2}(k+2)(2 q(X)-4)+\frac{1}{2} L^{2},
$$

We get $L^{2} \leq 4 k+6$. Therefore $L^{2}=4 k+5$ or $4 k+6$.

(III-B-1) The case in which $L^{2}=4 k+6$.

Then $f$ is the relatively minimal elliptic fibration, $f$ has no multiple fiber, and $q(X)=g(C)+1$. In particular, $f$ is a locally trivial fibration. Since $\kappa(X)=1$, we get that $q(X) \geq 3$ by the canonical bundle formula. This is the type (2) in Theorem 3.1.

(III-B-2) The case in which $L^{2}=4 k+5$.

Since $L^{2}$ is odd, $f$ is not relatively minimal. By Lemma 1.10 , we have $L F \geq 2 k+2$. Hence $K_{X} L \geq(2 k+2)(2 q(X)-4)+1$ and we get $g(L) \geq$ $(k+2) q(X)+k q(X)-2 k$. Since $q(X) \geq 3, g(L)>(k+2) q(X)$ is obtained and this is a contradiction.

This completes the proof of Theorem 3.1.

§4. The case in which $\kappa(X)=2$

THEOREM 4.1. Let $(X, L)$ be a polarized surface with $\kappa(X)=2$. Assume that $\mathrm{Bs}|L|=\emptyset$ and $h^{0}(L) \geq 5$. Then $g(L) \geq 2 q(X)$.

Proof. First we prove the following Claim;

Claim 4.2. Let $x_{1} \in X$ be a point and let $\psi: X^{\prime} \rightarrow X$ be blowing up at $x_{1}$. We put $L_{1}:=\psi^{*} L-E$ and $L_{2}:=L_{1}-E$, where $E$ is the (-1)-curve of $\psi$. Then $h^{0}\left(L_{2}\right) \geq 2$.

Proof of Claim 4.2. By the following exact sequence

$$
0 \rightarrow H^{0}\left(L_{1}\right) \rightarrow H^{0}\left(\psi^{*} L\right) \rightarrow H^{0}\left(\mathcal{O}_{E}\right),
$$

we get that $h^{0}(L)-h^{0}\left(L_{1}\right) \leq h^{0}\left(\mathcal{O}_{E}\right)=1$.

By the following exact sequence

$$
0 \rightarrow H^{0}\left(L_{2}\right) \rightarrow H^{0}\left(L_{1}\right) \rightarrow H^{0}\left(\mathcal{O}_{E}(1)\right),
$$

we get that $h^{0}\left(L_{1}\right)-h^{0}\left(L_{2}\right) \leq h^{0}\left(\mathcal{O}_{E}(1)\right)=2$.

Hence $h^{0}\left(L_{2}\right) \geq 2$. 
Let $\Lambda_{2} \subset\left|L_{2}\right|$ be a linear pencil and let $\Lambda:=\psi_{*} \Lambda_{2}$. Then $\Lambda \subset\left|L \otimes m_{x_{1}}^{2}\right|$, where $m_{x_{1}}$ is the ideal sheaf of $x_{1}$. Let $\Lambda_{M}$ be the movable part of $\Lambda$ and let $Z$ be the fixed part of $\Lambda$.

(I) The case in which $Z=0$.

In this case, $\operatorname{dim} \operatorname{Bs} \Lambda \leq 0$. Since $x_{1} \in \mathrm{Bs} \Lambda$, we get mult $x_{x_{1}} D \geq 2$ for any $D \in \Lambda$. Here we use the same argument as in the proof of Theorem 3.1 in [Fk3].

Let $\varphi$ be a rational map $X \rightarrow P^{1}$ associated with $\Lambda_{M}$, let $\mu: X^{\prime} \rightarrow X$ be an elimination of indeterminacy of $\varphi$, and let $\varphi^{\prime}$ be the morphism $X^{\prime} \rightarrow \boldsymbol{P}^{1}$.

Let $\varphi^{\prime}=\delta \circ f: X \rightarrow C \rightarrow \boldsymbol{P}^{1}$ be its Stein factorization, where $\delta: C \rightarrow \boldsymbol{P}^{1}$ is a finite morphism and $f$ is a fiber space $X \rightarrow C$.

We put $a:=\operatorname{deg} \delta, L^{\prime}:=\mu^{*} L$, and $F_{f}$ is a general fiber of $f$.

Let $b$ be the number of times of blowing up $\mu$.

We put $\mu=\mu_{1} \circ \mu_{2} \circ \cdots \circ \mu_{b}: X^{\prime}=X_{b} \rightarrow X_{b-1} \rightarrow \cdots \rightarrow X_{1} \rightarrow X_{0}=X$, where $\mu_{i}$ is the blowing down of $(-1)$-curve $E_{l}$ and $\mu_{1}: X_{1} \rightarrow X_{0}$ is the blowing up at $x_{1} \in X$. Let $L_{0}=L$ and $L_{l}=\mu_{i}^{*} L_{l-1}$ for $i=1,2, \ldots, b$. Then $L^{\prime}=L_{b}$. We take an element $M$ of $\Lambda_{M}$. We put $M_{0}=M$ and $\Lambda_{M}=\Lambda_{0}$. Let $\Lambda_{l}$ be the movable part of $\mu_{i}^{*} \Lambda_{l-1}$. Then we write $\Lambda_{l}=\mu_{i}^{*} \Lambda_{l-1}-n_{i} E_{l}$, where $n_{i}>0$ for $i=1, \ldots, b$. Let $M_{l}=\mu_{i}^{*} M_{l-1}-n_{i} E_{l}$ and $M^{\prime}=M_{b}$. Then $M_{l} \in \Lambda_{l}$. We remark that $M^{\prime} \equiv a F_{f}$, where $\equiv$ is numerical equivalence.

Then

$$
\left(K_{X^{\prime}}+L^{\prime}\right)\left(L^{\prime}-M^{\prime}\right)=\left(K_{X}+L\right)(L-M)-\sum_{i=1}^{b} n_{i} .
$$

Since $M^{\prime} \equiv a F_{f}$, then

$$
M^{2}=\sum_{i=1}^{b} n_{i}^{2}
$$

We remark that $K_{X}+L$ is nef. By construction, $L-M$ is an effective divisor. Hence $\left(K_{X}+L\right)(L-M) \geq 0$. Because $n_{i}>0$ and $n_{1} \geq 2$, we have

$$
2+\sum_{i=1}^{b} n_{i} \leq \sum_{i=1}^{b} n_{i}^{2}
$$

Therefore by the above

$$
\begin{aligned}
\left(K_{X^{\prime}}+L^{\prime}\right) L^{\prime} & =\left(K_{X^{\prime}}+L^{\prime}\right) M^{\prime}+\left(K_{X}+L\right)(L-M)-\sum_{i=1}^{b} n_{i} \\
& \geq\left(K_{X^{\prime}}+L^{\prime}\right) M^{\prime}-\sum_{l=1}^{b} n_{i}^{2}+2 \\
& =\left(K_{X^{\prime}}+L^{\prime}\right) M^{\prime}-M^{2}+2 \\
& =K_{X^{\prime}} M^{\prime}+L M-M^{2}+2 .
\end{aligned}
$$


On the other hand, $L M-M^{2}=(L-M) M$. Since $M \in \Lambda_{M}, M$ is a nef divisor on $X$. So we have $L M-M^{2} \geq 0$.

Hence

$$
\begin{aligned}
\left(K_{X^{\prime}}+L^{\prime}\right) L^{\prime} & \geq K_{X^{\prime}} M^{\prime}+2 \\
& =2 a\left(g\left(F_{f}\right)-1\right)+2 \\
& \geq 2 g\left(F_{f}\right)
\end{aligned}
$$

by $a \geq 1$.

Therefore

$$
\begin{aligned}
g\left(L^{\prime}\right) & \geq g\left(F_{f}\right)+1 \\
& =2 \frac{g\left(F_{f}\right)+1}{2} \\
& \geq 2 q\left(X^{\prime}\right)
\end{aligned}
$$

by Theorem 1 in $[\mathrm{X}]$. Since $g(L)=g\left(L^{\prime}\right)$ and $q(X)=q\left(X^{\prime}\right)$, we obtain $g(L) \geq$ $2 q(X)$.

(II) The case in which $Z \neq 0$.

Let $M \in \Lambda_{M}$. We remark that $M Z>0$ because $M+Z \in|L|$ is 1 -connected.

(II-1) $M^{2}>0$ case.

Then $M$ is nef-big and $\operatorname{dim} \operatorname{Bs}|M| \leq 0$. So we get

$$
\begin{aligned}
g(L) & =1+\frac{1}{2}\left(K_{X}+L\right) L \\
& \geq 1+\frac{1}{2}\left(K_{X}+L\right) M \\
& =1+\frac{1}{2}\left(K_{X}+M\right) M+\frac{1}{2} M Z \\
& \geq 1+\frac{1}{2}\left(K_{X}+M\right) M+\frac{1}{2} \\
& =g(M)+\frac{1}{2}
\end{aligned}
$$

since $M Z>0$. On the other hand $g(M) \geq 2 q(X)-1$ by Corollary 3.2 in [Fk3]. Since $g(L) \in Z$, we get $g(L) \geq 2 q(X)$.

(II-2) $M^{2}=0$ case.

Then $\mathrm{Bs} \Lambda_{M}=\emptyset$. Let $\varphi: X \rightarrow \boldsymbol{P}^{1}$ be a surjective morphism defined by $\Lambda_{M}$. By taking Stein factorization, if necessary, there exists a smooth curve $C$, a finite morphism $\pi: C \rightarrow \boldsymbol{P}^{1}$, and a surjective morphism with connected fibers $f: X \rightarrow C$ such that $\varphi=\pi \circ f$.

(II-2-1) The case in which $g(C)=0$.

Then we can prove that $g(L) \geq g(M)+(1 / 2)$ by the same argument as above. On the other hand by construction we have $M \equiv a F$, where $a$ is a 
natural number. Hence $g(L) \geq g(F)+(1 / 2) \geq 2 q(X)-(1 / 2)$ by Theorem 1 in [X]. So we get $g(L) \geq 2 q(X)$.

(II-2-2) The case in which $g(C) \geq 1$.

Then $a \geq 2$ by construction. We remark that $L F \geq 2$ for a fiber $F$ of $f$ because Bs $|L|=\emptyset$ and $\kappa(X)=2$. Since $K_{X / C}+L$ is nef by Lemma 1.15 (3),

$$
\begin{aligned}
g(L) & =g(C)+\frac{1}{2}\left(K_{X / C}+L\right) L+(L F-1)(g(C)-1) \\
& \geq 2 g(C)-1+\frac{1}{2}\left(K_{X / C}+L\right)(a F)+\frac{1}{2}\left(K_{X / C}+L\right) Z \\
& \geq 2 g(C)-1+2 g(F)-2+2 \\
& =2(g(C)+g(F))-1 .
\end{aligned}
$$

On the other hand by Lemma 1.16 we get $q(X) \leq g(C)+g(F)$.

If $g(C)+g(F) \geq q(X)+1$, then $g(L)>2 q(X)$. So it is sufficient to consider the case in which $q(X)=g(C)+g(F)$. Then by Lemma 1.16, we get $X \sim_{\text {bir }} F \times C . \quad$ By Lemma 1.18, we get $g(L) \geq 2 q(X)$. This completes the proof of Theorem 4.1.

COROLlaRY 4.3. Let $(X, L)$ be a polarized surface with $\kappa(X)=2$. If $L$ is very ample, then $g(L) \geq 2 q(X)$.

Proof. Since $L$ is very ample and $\kappa(X)=2$, we get $h^{0}(L) \geq 4$.

If $h^{0}(L) \geq 5$, then $g(L) \geq 2 q(X)$ by Theorem 4.1 .

If $h^{0}(L)=4$, then $X$ is a hypersurface in $P^{3}$. Hence we get that $q(X)=0$ and so we have $g(L)>2 q(X)$.

THEOREM 4.4. Let $(f, X, C, L)$ be a polarized fiber space with $\operatorname{dim} X=2$ and $\kappa(X)=2$. If $L$ is $k$-very ample with $k \geq 0$ and $q(X) \leq g(C)+1$, then $g(L) \geq(k+2) q(X)$.

Proof. (I) The case in which $k=0$.

(I-1) The case in which $g(C)=0$.

Then $q(X) \leq 1$. So we get $g(L) \geq 2 \geq 2 q(X)$. Then

(I-2) The case in which $g(C) \geq 1$.

$$
\begin{aligned}
g(L) & =g(C)+\frac{1}{2}\left(K_{X / C}+L\right) L+(L F-1)(g(C)-1) \\
& \geq 2 g(C)-1+\frac{1}{2}\left(K_{X / C}+L\right) L
\end{aligned}
$$

since $L F \geq 2$ by Lemma 1.9 , where $F$ is a general fiber of $f$.

(I-2-1) The case in which $L^{2} \leq 3$. 
If $L^{2} \leq 2$, then by Proposition 1.3 we get $q(X)=0$ and so we have $g(L)>2 q(X)$. If $L^{2}=3$ and $K_{X / C} L \geq 2$, then $g(L) \geq 2 g(C)+(3 / 2)$ and we get $g(L) \geq 2 g(C)+2 \geq 2 q(X)$.

If $L^{2}=3$ and $K_{X / C} L \leq 1$, then $0 \leq K_{X^{\prime} / C} L^{\prime} \leq K_{X / C} L \leq 1$, where $f^{\prime}: X^{\prime} \rightarrow C$ is the relatively minimal model of $f, \mu: X \rightarrow X^{\prime}$ is its birational morphism, and $L^{\prime}=\mu_{*}(L)$ in the sense of cycle theory. Since $\left(L^{\prime}\right)^{2} \geq 3$, we get $\left(K_{X^{\prime} / C}\right)^{2}=0$ by Hodge index Theorem and Lemma 1.15. In particular $f^{\prime}$ is a locally trivial fibration.

If $K_{X^{\prime} / C} L^{\prime}=0$, then $\left(K_{X^{\prime} / C}\right)^{2}=0$ and $K_{X^{\prime} / C} \equiv 0$. But this is impossible because $\kappa(X)=2$. If $K_{X^{\prime} / C} L^{\prime}=1$, then $K_{X / C} L=K_{X^{\prime} / C} L^{\prime}$ and so we get $X \cong X^{\prime}$. In particular, $f$ is a locally trivial fibration. By Lemma 1.17 we get $g(L) \geq 2 q(X)$.

(I-2-2) The case in which $L^{2} \geq 4$.

By Lemma 1.15 , we get $K_{X / C} L \geq 0$.

If $K_{X / C} L=0$, then $X$ is minimal, $\left(K_{X / C}\right)^{2}=0$, and $K_{X / C} \equiv 0$. But this is impossible because $\kappa(X)=2$. Hence $K_{X / C} L \geq 1$. So we get $g(L) \geq 2 g(C)+$ (3/2). Therefore $g(L) \geq 2 g(C)+2 \geq 2 q(X)$.

(II) The case in which $k=1$.

(II-1) $g(C)=0$ case.

Then $q(X) \leq 1$. Since $L$ is very ample and $\kappa(X)=2$, we get $L^{2} \geq 2$. Hence $g(L) \geq 3$. So we get that $g(L) \geq 3 q(X)$.

(II-2) $g(C) \geq 1$ case.

By Lemma 1.9 , we get that $L F \geq 4$ because $g(F) \geq 2$.

(II-2-1) The case in which $L^{2} \leq 8$.

We remark that $L^{2} \geq 2$ since $\kappa(X)=2$. Hence $g(L) \geq 3$.

If $h^{0}(L)=4$, then $X$ is a hypersurface in $P^{3}$. Hence $q(X)=0$ and we get $g(L)>3 q(X)$.

If $h^{0}(L) \geq 6$ and $L^{2} \leq 7$, then $L^{2} \geq 2 \Delta(L)+1$ and $g(L) \geq 3 \geq \Delta(L)$. Hence by Theorem 1.2 this is impossible.

If $h^{0}(L) \geq 6$ and $L^{2}=8$, then $\Delta(L) \leq 4$. Since $L^{2}=8$, we get $g(L) \geq 6>$ $\Delta(L)$. If $\Delta(L)=4$, then by Theorem 1.2 we get $g(L)>3 q(X)$. If $\Delta(L) \leq 3$, then $L^{2} \geq 2 \Delta(L)+1$ and this is impossible by Theorem 1.2 .

If $h^{0}(L)=5$ and $L^{2} \leq 5$, then $g(L) \geq 2 \geq \Delta(L)$ and $L^{2} \geq 2 \Delta(L)+1$. But by Theorem 1.2, this is impossible.

If $h^{0}(L)=5$ and $L^{2}=6$, then $g(L) \geq 5>3=\Delta(L)$ and $L^{2}=2 \Delta(L)$. Hence by Theorem 1.2 we get $g(L)>3 q(X)$.

If $h^{0}(L)=5$ and $L^{2}=7$, then by Proposition 1.19 we get $g(L) \leq 6$. Hence $K_{X} L \leq 3$. Let $\mu: X \rightarrow X^{\prime}$ be the minimal of $X$ and $L^{\prime}:=\mu_{*}(L)$. Then $3 \geq$ $K_{X} L \geq K_{X^{\prime}} L^{\prime}$ and $\left(L^{\prime}\right)^{2} \geq L^{2}=7$. Hence $K_{X^{\prime}}^{2} \leq 1$ by Hodge index Theorem. By Proposition 1.13, we get $q(X)=0$ and $g(L)>3 q(X)$.

If $h^{0}(L)=5$ and $L^{2}=8$, then by Proposition 1.19 we get $g(L) \leq 9$. Hence $K_{X} L \leq 8$. Let $\mu: X \rightarrow X^{\prime}$ be the minimal of $X$ and $L^{\prime}:=\mu_{*}(L)$. Then we remark that $K_{X} L \geq K_{X^{\prime}} L^{\prime}$ and $\left(L^{\prime}\right)^{2} \geq L^{2}$. Since $K_{X^{\prime}}^{2}>0$ and $L^{2}$ is even, we get $K_{X} L \geq 4$ by Hodge index Theorem.

If $K_{X} L=4$, then $K_{X^{\prime}}^{2} \leq 2$ and $q(X) \leq 1$ by Proposition 1.13. Hence $g(L)=7>3 q(X)$. 
If $K_{X} L=6$, then $K_{X^{\prime}}^{2} \leq 4$ and $q(X) \leq 2$ by Proposition 1.13. Hence $g(L)=8>3 q(X)$.

If $K_{X} L=8$, then $K_{X^{\prime}}^{2} \leq 8$ and $q(X) \leq 4$ by Proposition 1.13. If $q(X) \leq 3$, then $g(L)=9 \geq 3 q(X)$. So we may assume $q(X)=4$. In this case we get $K_{X^{\prime}}^{2}=8$ and so we obtain $X=X^{\prime}$ and $K_{X} \equiv L$ by Hodge index Theorem. By Proposition 1.6 we get $\chi\left(\mathcal{O}_{X}\right)=6$. Therefore $p_{g}=9$. But by Proposition 1.13 this is a contradiction because $K_{X}^{2}=8$.

Then

(II-2-2) The case in which $L^{2} \geq 9$.

$$
\begin{aligned}
g(L) & =g(C)+\frac{1}{2}\left(K_{X / C}+L\right) L+(L F-1)(g(C)-1) \\
& \geq 3 g(C)+g(C)+\frac{3}{2} \\
& \geq 3 g(C)+\frac{5}{2} .
\end{aligned}
$$

Hence $g(L) \geq 3 g(C)+3 \geq 3 q(X)$.

(III) The case in which $k \geq 2$.

Then by Theorem 1.7 , we get $L^{2} \geq 4 k+5$.

If $g(C) \geq 1$, then we get

$$
\begin{aligned}
g(L) & =g(C)+\frac{1}{2}\left(K_{X / C}+L\right) L+(L F-1)(g(C)-1) \\
& \geq(k+3) g(C)+k+\frac{1}{2} \\
& =(k+2)(g(C)+1)+g(C)-\frac{3}{2}
\end{aligned}
$$

since $L F \geq k+3$ and $K_{X / C} L \geq 0$ by Lemma 1.9 and Lemma 1.15.

Hence $g(L) \geq(k+2)(g(C)+1)+g(C)-1 \geq(k+2) q(X)$.

If $g(C)=0$, then $q(X) \leq 1$. So we get

$$
\begin{aligned}
g(L) & \geq 1+\frac{1}{2}(1+4 k+5) \\
& =2 k+4 \\
& >(k+2) q(X)
\end{aligned}
$$

This completes the proof of Theorem 4.4 .

Corollary 4.5. Let $(f, X, C, L)$ be a polarized fiber space with $\operatorname{dim} X=2$ and $\kappa(X)=2$. Assume that $g(F)=2$ for a general fiber $F$ of $f$ and $L$ is $k$-very ample with $k \geq 0$. Then $g(L) \geq(k+2) q(X)$.

Proof. By Lemma 1.16, we get $q(X) \leq g(C)+2$.

If $q(X)=g(C)+2$, then by Lemma 1.16 and Lemma 1.18 we get the assertion. 
If $q(X) \leq g(C)+1$, then by Theorem 4.4 we get the assertion.

Appendix. Let $(X, L)$ be a polarized surface with $\kappa(X)=-\infty$. Assume that $L$ is $k$-very ample. In this appendix, we consider a lower bound for sectional genus with $\kappa(X)=-\infty$.

If $q(X)=0$, then $g(L) \geq(k+2) q(X)$. So we assume that $q(X) \geq 1$.

If $(X, L)$ is not a scroll over a smooth curve $C$, then we can prove that $g(L) \geq 2 q(X)$ for any polarized surface with $\kappa(X)=-\infty$.

Here we consider the case in which $k \geq 2$.

Lemma A.1. Let $(X, L)$ be a polarized surface. Assume that $\kappa(X)=-\infty, L$ is $k$-very ample with $k \geq 2$, and $L^{2} \leq 4 k+4$. Then $g(L) \geq(k+2) q(X)$ unless

(*) $k=2, X$ is a $\boldsymbol{P}^{1}$-bundle over a smooth curve $C$ of genus two, and $L \equiv 2 C_{0}+2 F$ with $C_{0}^{2}=2$, where $C_{0}$ is a minimal section of the projection map $X \rightarrow C$ and $F$ is its fiber.

Proof. By the classification of $(X, L)$ with $L^{2} \leq 4 k+4$ by Di Rocco [Di], we obtain the assertion.

We remark that if $(X, L)$ is $(*)$, then $g(L)=7$.

THeOREM A.2. Let $(X, L)$ be a polarized surface with $\kappa(X)=-\infty$ and $q(X) \geq 1$. Assume that $X$ is relatively minimal, and $L$ is $k$-very ample with $k \geq 2$. Then $g(L) \geq k q(X)$.

Proof. Let $f: X \rightarrow C$ be the $\boldsymbol{P}^{1}$-bundle. Let $\mathscr{E}$ be a normalized vector bundle of rank two on $C$ such that $X=\boldsymbol{P}(\mathscr{E})$, and let $C_{0}$ be a minimal section of $f$. We can write $L \equiv a C_{0}+b F$, where $F$ is a fiber of $f$. Let $e:=-C_{0}^{2}$. Then

$$
g(L)=a g(C)+(a-1)\left(b-\frac{1}{2} a e-1\right) .
$$

If $e \geq 0$, then by Proposition 2.20 in [Ha], we get $b-a e>0$. Hence $b-(1 / 2) a e-1>(1 / 2) a e-1 \geq-1$. On the other hand, $a \geq k$ by Lemma 1.9. Therefore we get $g(L) \geq k g(C)=k q(X)$.

If $e<0$, then by Proposition 2.21 in [Ha], we get $b-(1 / 2) a e>0$. If $b-(1 / 2) a e \geq 1$, then $g(L) \geq k q(X)$ by the same argument as above. If $b-(1 / 2) a e=1 / 2$, then $L^{2}=a(2 b-a e)=a$. By Lemma A.1 we may assume that $a=L^{2} \geq 4 k+5$. Hence

$$
\begin{aligned}
g(L) & =\frac{a+1}{2} g(C)+\frac{a-1}{2} g(C)-\frac{a-1}{2} \\
& \geq(2 k+3) g(C)+\frac{a-1}{2}-\frac{a-1}{2} \\
& \geq(2 k+3) q(X) .
\end{aligned}
$$

This completes the proof of Theorem A.2. 
THEOREM A.3. Let $(X, L)$ be a polarized surface with $\kappa(X)=-\infty$ and $q(X) \geq 1$. Assume that $X$ is not relatively minimal, and $L$ is $k$-very ample with $k \geq 2$. Then $g(L) \geq(k+2) q(X)$.

Proof. Let $f: X \rightarrow C$ be the Albanese fibration, where $C$ is a smooth curve of genus $g(C)=q(X) \geq 1$. We remark that

$$
g(L)=g(C)+\frac{1}{2}\left(K_{X / C}+L\right) L+(L F-1)(g(C)-1)
$$

where $F$ is a general fiber of $f$.

By assumption $L F \geq 2 k$ by Lemma 1.9. So we can prove that $\kappa\left(K_{F}+(1 / 2) L_{F}\right) \geq 0$ for a general fiber $F$ of $f$. By Lemma 0.1 in [Fk4], we get $\left(K_{X / C}+(1 / 2) L\right) L \geq 0$. Therefore

$$
\begin{aligned}
g(L) & \geq g(C)+\frac{1}{4} L^{2}+(2 k-1)(g(C)-1) \\
& =2 k g(C)+\frac{1}{4} L^{2}-(2 k-1) \\
& =(k+2) g(C)+(k-2) g(C)+\frac{1}{4} L^{2}-(2 k-1) .
\end{aligned}
$$

By Lemma A.1, we may assume that $L^{2} \geq 4 k+5$. Hence

$$
\begin{aligned}
g(L) & \geq(k+2) g(C)+(k-2)+k+\frac{5}{4}-(2 k-1) \\
& =(k+2) g(C)+\frac{1}{4} .
\end{aligned}
$$

So we obtain the assertion.

\section{REFERENCES}

[ACGH] E. Arbarello, M. Cornalba, P. Gri\%oths and J. Harris, Geometry of Algebraic Curves, Volume I, Springer-Verlag, 1985.

[BaSo] E. BAllico AND A. J. Sommese, Projective surfaces with $k$-very ample line bundles of degree $\leq 4 k+4$, Nagoya Math. J., 136 (1994), 57-79.

[Bea] A. Beauville, L'inegalite $p_{g} \geq 2 q-4$ pour les surfaces de type général, Bull. Soc. Math. France, 110 (1982), 343-346.

[BeSo1] M. C. Beltrametri AND A. J. Sommese, On the preservation of $k$-very ampleness under adjunction, Math. Z., 212 (1993), 257-283.

[BeSo2] M. C. Beltrametri AND A. J. Sommese, The Adjunction Theory of Complex Projective Varieties, de Gruyter Expositions in Mathematics, 16, Walter de Gruyter, Berlin, New York, 1995.

[Bes] G. M. Besana, On polarized surfaces of degree three whose adjoint bundles are not spanned, Arch. Math. (Basel), 65 (1995), 161-167

[De] O. DebarRe, Inégalités numériques pour les surfaces de type général, Bull. Soc. Math. France, 110 (1982), 319-346; Addendum, Bull. Soc. Math. France, 111 (1983), 301-302. 
[Di] S. Di Rocco, Projective surfaces with $k$-very ample line bundles of genus $\leq 3 k+1$, Manuscripta Math., 91 (1996), 35-59.

[Fj1] T. FuנITA, On hyperelliptic polanzed vaneties, Tohoku Math. J., 35 (1983), 1-44.

[Fj2] T. FuITA, Classification Theories of Polarized Varneties, London Math. Soc. Lecture Note Ser., 155, Cambridge Univ. Press, 1990.

[Fk1] Y. FuKUma, A lower bound for the sectional genus of quast-polarized surfaces, Geom. Dedicata, 64 (1997), 229-251.

[Fk2] Y. FukUmA, A lower bound for the sectional genus of quasi-polanzed surfaces II, to appear in Rend. Sem. Mat. Univ. Politec. Torino.

[Fk3] Y. FuKUMA, On sectional genus of quasi-polanzed manifolds with non-negative Kodarra dimension, Math. Nachr., 180 (1996), 75-84.

[Fk4] Y. FuKUMA, A lower bound for sectional genus of quasi-polarized manifolds II, preprint.

[Fk5] Y. FUKUMA, On $d$-very ample line bundles on abelian surfaces, preprint.

[Ha] R. HARTSHORNE, Algebraic Geometry, Graduate Texts in Math., 52, Springer-Verlag, 1977

[LB] H. Lange ANd Ch. Birkenhake, Complex Abelian Varieties, Grundlehren Math. Wiss. 302, Springer-Verlag, 1992.

[La] R. LAZARSFELD, A Barth-type theorem for branched coverngs of projective space, Math. Ann., 249 (1980), 153-162.

[Pe] U. Persson, Chern invariants of surfaces of general type, Compositio Math., 43 (1981), 3-58.

[Se] F. SERRANo, Elliptic surfaces with an ample divisor of genus two, Pacific J. Math., 152 (1992), 187-199.

[X] G. XIAO, Irregularity of surfaces with a linear pencil, Duke Math. J., 55 (1987), $597-$ 602.

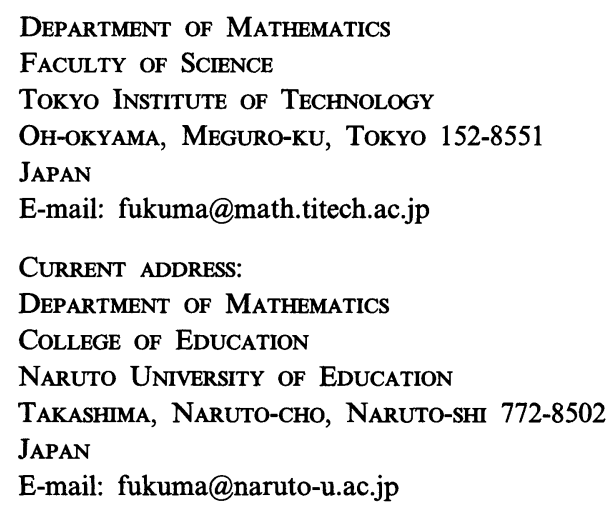

Note added in proof. After the acceptance of this paper for publication, Dr. Kazuyoshi Takahashi pointed out to the author that the type $(*)$ in Lemma A. 1 is excluded because $L^{2}=16>4 k+5$ and this contradicts the assumption of Lemma A.1. But we need not to change the assertion of Theorem A.2 and Theorem A.3. The author would like to thank Dr. K. Takahashi for pointing out these. 